\title{
Erratum: Changes in circulating microRNAs after radiochemotherapy in head and neck cancer patients
}

Isolde Summerer ${ }^{1}$, Maximilian Niyazi ${ }^{2}$, Kristian Unger ${ }^{1,4}$, Adriana Pitea ${ }^{1}$, Verena Zangen ${ }^{1,4}$, Julia Hess ${ }^{1,4}$, Michael J Atkinson ${ }^{3}$, Claus Belka ${ }^{2,4}$, Simone Moert ${ }^{3}$ and Horst Zitzelsberger ${ }^{1,4^{*}}$

\section{Erratum}

After the publication of this work [1], a few points were brought to our attention:

The clinical data of patient 2 are incorrect. A revised version of Table 1 [1] showing the corrected patient characteristics is presented here. Consequently, the description of the patient cohort in the Materials and Methods section is incorrect. The correct description is: "Plasma miRNA analysis was performed on 18 HNSCC patients treated with local X-ray-irradiation using a linear accelerator [...].

17 out of 18 patients received concurrent chemotherapy (13 patients received 5-fluorouracil (5-FU) plus mitomycin $C(M M C)$ [21], 3 patients $M M C$ and 1 patient cisplatin weekly)".

There is a typing error in the Results section [1] concerning the $\mathrm{p}$ value of the up-regulation of $m i R-425-5 p$. The correct sentence is:

"The qRTPCR assays confirmed an up-regulation of miR-425-5p with a $p$ value close to the significance level $(p=0.052)[\ldots] "$.

The corrections do not affect any results or conclusions of the published work.

\footnotetext{
* Correspondence: zitzelsberger@helmholtz-muenchen.de

${ }^{1}$ Research Unit Radiation Cytogenetics, Helmholtz Center Munich, Ingolstaedter Landstr 1, 85764 Neuherberg, Germany

${ }^{4}$ Clinical Cooperation Group 'Personalized Radiotherapy of Head and Neck

Cancer', Helmholtz Center Munich, Ingolstaedter Landstr 1, 85764

Neuherberg, Germany

Full list of author information is available at the end of the article
} 
Table 1 Patient characteristics

\begin{tabular}{|c|c|}
\hline Characteristic & Number of patients \\
\hline \multicolumn{2}{|l|}{ Gender } \\
\hline Male & 14 \\
\hline Female & 4 \\
\hline Median age, years & 57.5 \\
\hline Age range, years & $45.1-80.6$ \\
\hline \multicolumn{2}{|l|}{ Tumor site } \\
\hline Larynx & 5 \\
\hline Oropharynx & 3 \\
\hline Mouth floor & 2 \\
\hline Tongue & 2 \\
\hline Hypopharynx & 1 \\
\hline Maxilla & 1 \\
\hline Nasopharynx & 1 \\
\hline Sinuses & 1 \\
\hline Soft palate & 2 \\
\hline \multicolumn{2}{|l|}{ T-Stage } \\
\hline I & 4 \\
\hline$\|$ & 3 \\
\hline III & 5 \\
\hline IV & 6 \\
\hline \multicolumn{2}{|l|}{ N-Stage } \\
\hline NO & 3 \\
\hline $\mathrm{N} 1$ & 4 \\
\hline N2 & 11 \\
\hline \multicolumn{2}{|l|}{ M-Stage } \\
\hline MO & 17 \\
\hline M1 & 1 \\
\hline \multicolumn{2}{|c|}{$\begin{array}{l}\text { Concomitant therapy } \\
\text { (in addition to radiotherapy) }\end{array}$} \\
\hline $5-F U+M M C$ & 13 (patient 2, 3, 5-7, 9-13, 15-17) \\
\hline MMC & 3 (patient $1,14,18$ ) \\
\hline Cisplatin & 1 (patient 8) \\
\hline Cetuximab & 1 (patient 4) \\
\hline \multicolumn{2}{|l|}{ Acute toxicity } \\
\hline Severe & 12 \\
\hline Moderate & 5 \\
\hline n.a. & 1 \\
\hline
\end{tabular}

5-FU = 5-fluorouracil; $M M C=$ mitomycin $C ;$ n.a. $=$ not available.

\section{Author details}

${ }^{1}$ Research Unit Radiation Cytogenetics, Helmholtz Center Munich, Ingolstaedter Landstr 1, 85764 Neuherberg, Germany. ${ }^{2}$ Department of Radiation Oncology, University of Munich, Marchioninistr 15, 81377 Munich Germany. ${ }^{3}$ Institute of Radiation Biology, Helmholtz Center Munich, Ingolstaedter Landstr 1, 85764 Neuherberg, Germany. ${ }^{4}$ Clinical Cooperation Group 'Personalized Radiotherapy of Head and Neck Cancer', Helmholtz Center Munich, Ingolstaedter Landstr 1, 85764 Neuherberg, Germany.
Received: 25 March 2015 Accepted: 25 March 2015

Published online: 24 April 2015

\section{Reference}

1. Summerer I, Niyazi M, Unger K, Pitea A, Zangen V, Hess J, et al. Changes in circulating microRNAs after radiochemotherapy in head and neck cancer patients. Radiat Oncol. 2013;8:296. 